\title{
Late onset spondylarthropathy: clinical and biological comparison with early onset patients
}

\author{
Didier Caplanne, Florence Tubach, Jean Marie Le Parc
}

\begin{abstract}
Objective-To compare the clinical, radiological, and biological profile of patients presenting late onset spondylarthropathy (LOSPA) with patients with early onset spondylarthropathy (EOSPA). Methods-During the period April 1987 to April 1995 a retrospective chart review of inpatients and outpatients identified eight patients with LOSPA. They were matched with 32 patients with EOSPA examined during the same period of time. Clinical, radiological, and biological signs were compared. All patients fulfilled Amor criteria for spondylarthropathy.
\end{abstract}

Results-Mean age of patients with LOSPA was 65.1 years (range 58-72), and 26.6 years (range 11-40) in patients with EOSPA. The sex ratio (female/male) was $5 / 3$ in LOSPA and 9/23 in EOSPA ( $p=$ 0.007). Patients with LOSPA had more significantly cervical and dorsal pain ( $p=$ $0.002, p=0.02$ respectively), anterior chest wall involvement $(p=0.04)$, number of peripheral arthritis $(p=0.04)$, aseptic osteitis $(p=0.004)$, and systemic symptoms : fever, fatigue, weight loss $(p=$ 0.04). Mean (SD) erythrocyte sedimentation rate was 87 (24) in LOSPA and 24 (35) in EOSPA patients $(p=0.001)$. Inflammatory bowel disease was diagnosed in three patients with EOSPA. A definite family history of SPA was found in $50 \%$ of patients with LOSPA and in $31 \%$ of patients with EOSPA. A clear response to NSAID was obtained in $62 \%$ of LOSPA patients and in $90.6 \%$ of EOSPA patients $(p=0.05)$. Three LOSPA patients (two with Crohn's disease) not responding to NSAID were successfully treated with prednisone.

Conclusion-The onset of spondylarthropathy is uncommon after 55 years. Patients with LOSPA, according to accepted international criteria present a different clinical and biological profile when compared with younger patients. These results suggests that age may influence the presentation of SPA at onset.

(Ann Rheum Dis 1997;56:176-179)

The spondylarthropathies (SPAs) are a group of related disorders including ankylosing spondylitis, reactive arthritis, psoriatic arthritis, forms related to inflammatory bowel disease, and a group of undifferentiated forms.
SPA usually begins in young or middle aged adults. ${ }^{1}$ Very few studies have described patients with classic late onset SPA. ${ }^{2-5}$

Dubost and Sauvezie described an atypical presentation of SPA in older men. ${ }^{6}$ More recently, Olivieri and colleagues reported on a group of 23 patients with undifferentiated SPA in patients older than 45 years and not meeting criteria for the definite presentation of SPA. They found the same clinical spectrum of presentation as in children and adults. ${ }^{5}$

The aim of our study was to compare the clinical presentation of classic late onset SPA (LOSPA) with a group of young or middle age patients, early onset SPA (EOSPA).

\section{Patients and methods}

During the period April 1987 to April 1995 we identified by computer assisted search of inpatient and outpatient register, eight patients who were examined in the department of rheumatology for their first episode of inflammatory SPA after 55 years of age.

To examine possible differences in clinical and laboratory features between LOSPA and earlier onset we matched each of the eight patients with four patients with onset of SPA before the age of 40 and who were examined for their first episode of SPA and followed up during the same period of time.

All patients fulfilled the AMOR criteria for spondylarthropathy. ${ }^{7}$ Patients were examined within three months after the first onset of rheumatic symptoms. Careful history excluded pre-existing inflammatory symptoms of the spine and peripheral joints. Each patient whether referred or seen as a primary care patient was examined and followed up by a consultant rheumatologist. Spine and pelvic $x$ rays were obtained for the search of sacroiliitis, syndesmophytes, and discitis. In case of undetermined sacroiliitis on $x$ rays a computed tomogram was obtained to confirm the presence of sacroiliitis. A bone technetium scan was obtained when peripheral osteitis was suspected clinically.

To eliminate previous signs of inflammatory spondylarthropathy in the past years or during adolescence, the general practicioner and private rheumatologist of patients were contacted for medical chart review. Previous spine $x$ rays were also reviewed to eliminate pre-existing signs of SPA.

Inflammatory bowel disease was established after ileocolonoscopy confirmed typical macroscopic and histological aspects of Crohn's disease and ulcerative colitis.
Accepted for publication 17 December 1996. 


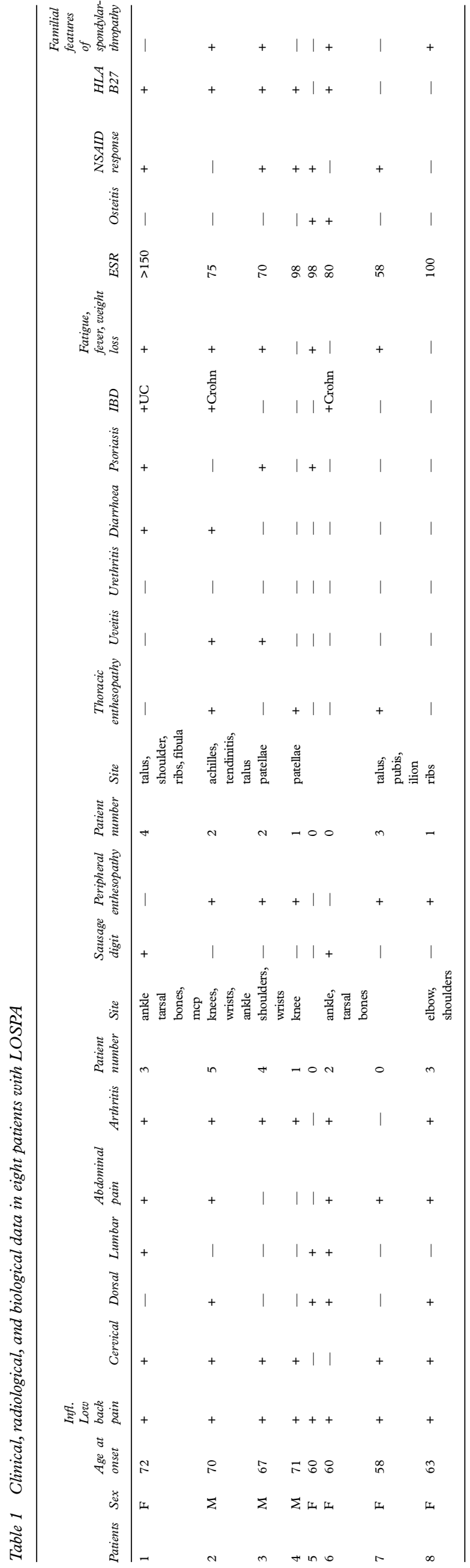

Inflammatory bowel disease was not systematically investigated in our patients. Ileocolonoscopy was performed (a) in the presence of persistent diarrhoea of three months duration or unexplained abdominal pain not resolving with symptomatic treatment, (b) when inflammatory bowel disease was present (with or without arthritis) in a first degree relative.

The clinical manifestations during onset and the first year of evolution including axial, peripheral, and extra-articular symptoms were obtained by chart review. Other recorded clinical information included erythrocyte sedimentation rate (ESR) at presentation, personal and family history, response to NSAID (that is, improvement of $>50 \%$ of inflammatory pain within 48 hours), and HLA B27 status.

STATISTICAL ANALYSIS

For comparison between LOSPA and EOSPA the $\chi^{2}$ test and Mann-Whitney test were applied. Significance was set at $\mathrm{p}<0.05$.

\section{Results}

Table 1 shows the clinical and laboratory data of the eight patients with LOSPA.

The mean (range) age at onset for the LOPSA and EOSPA groups were 65.1 (58-72) and 26.6 (11-40) respectively.

The sex ratio (female/male) for the LOPSA patients was $5 / 3$ and $9 / 23$ for the EOSPA $(\mathrm{p}=0.007)$.

Comorbidities were as follows: patient 1: hypertension treated with propanolol; patient 2: episodes of urolithiasis; patient 3: Dupuytren disease, myocardial infarction, treatment with calcium channel blockers; patient 4: glaucoma.

Table 2 lists the clinical manifestations at first examination and during the first year of evolution. Table 3 shows the extra-articular manifestations and associated disease.

Patients with late onset SPA had more frequent signs of inflammation of the cervical, dorsal spine and anterior chest wall. They also presented with more peripheral arthritis when compared with patient with younger onset of SPA. Aseptic osteitis was found in two patients with late onset SPA and in none of the other group of patients.

Systemic symptoms such as fever, weight loss, and fatigue were more frequent at initial

Table 2 Rheumatic manifestations in late onset and early onset spondylarthropathy groups during the first year of evolution

\begin{tabular}{llll}
\hline & $\begin{array}{l}\text { LOSPA } \\
(n=8)\end{array}$ & $\begin{array}{l}\text { EOSPA } \\
(n=32)\end{array}$ & $p$ Value \\
\hline Inflammatory spinal pain (\%) & 100 & 65.6 & 0.05 \\
$\quad$ cervical (\%) & 75 & 18.8 & 0.002 \\
$\quad$ dorsal (\%) & 62.5 & 21.9 & 0.02 \\
$\quad$ low back (\%) & 25 & 40.6 & $\mathrm{NS}$ \\
Abdominal pain (\%) & 62.5 & 62.5 & $\mathrm{NS}$ \\
Arthritis (\%) & 75 & 37.5 & $\mathrm{NS}$ \\
Number of arthritis patients & 2.3 & 1.5 & 0.04 \\
$\quad($ mean (SD)) & $(1.8)$ & $(3.9)$ & \\
Peripheral enthesopathy (\%) & 75 & 68.8 & $\mathrm{NS}$ \\
Number of enthesopathy & 1.6 & 2.5 & $\mathrm{NS}$ \\
$\quad$ patients (mean (SD)) & $(1.4)$ & $(2.6)$ & \\
Anterior chest wall involvement & 75 & 34.4 & 0.04 \\
$\quad$ (\%) & & & \\
Sausage digit (\%) & 25 & 15.6 & $\mathrm{NS}$ \\
Aseptic osteitis (\%) & 25 & 0 & 0.004 \\
\hline
\end{tabular}


Table 3 Extra-articular manifestations and associated diseases in late onset and early onset spondylarthropathy

\begin{tabular}{|c|c|c|c|}
\hline & $\begin{array}{l}\text { LOSPA } \\
(n=8)\end{array}$ & $\begin{array}{l}\text { EOSPA } \\
(n=32)\end{array}$ & p Value \\
\hline Acute uveitis $(\%)$ & 25 & 9.4 & NS \\
\hline Non-gonococcal urethritis or cervicitis $<1$ month before arthritis (\%) & 0 & 9 & NS \\
\hline Acute diarrhoea $<1$ month before arthritis $(\%)$ & 25 & 6.3 & NS \\
\hline Inflammatory bowel disease $(\%)$ & 37.5 & 6.3 & $\mathrm{P}=0.02$ \\
\hline Psoriasis $(\%)$ & 37.5 & 15.6 & NS \\
\hline $\mathrm{ESR}$ (mean (SD)) & $87(24)$ & $24(35)$ & $\mathrm{p}=0.001$ \\
\hline HLA B27 (\%) & 50 & 73 & NS \\
\hline
\end{tabular}

presentation in the LOSPA group (50\%) when compared with patients of the EOSPA group $(15.6 \%), p=0.04$. In addition the mean (SD) ESR was 87 (24) $\mathrm{mm}$ in the LOSPA patients and $24(35) \mathrm{mm}$ in the EOSPA patients $(\mathrm{p}=$ $0.001)$.

Inflammatory bowel disease was diagnosed at onset in three patients with LOSPA (two patients with Crohn's disease, one with ulcerative colitis) and in two patients with EOSPA.

A definite family history of SPA was made in four patients of the LOSPA group and in 10 patients in the EOSPA group. We were not able to find with sufficient confidence the exact age of onset of SPA in relatives of our patients. However none had their first symptoms after 40 years.

A clear response to NSAID was obtained in $62 \%$ of patients in the LOSPA compared with $90.6 \%$ in the patients with EOSPA $(\mathrm{p}=0.05)$. Two of three patients with LOSPA not responding to NSAID, were found to have Crohn's disease and were successfully treated with prednisone $30 \mathrm{mg} / \mathrm{day}$, and salazosulphapyridine.

In these patients ESR and $C$ reactive protein values returned to normal after six months while salazopyrine was still given with low dose corticosteroids ( $5 \mathrm{mg}$ prednisone daily).

Evolution in the remaining patients is as follows : one patient was successfully treated with indomethacine $75 \mathrm{mg}$ daily for three months then tapered at $25 \mathrm{mg}$. He has normal ESR and C reactive protein values in September 1996 with mild back pain but recurrence of inflammatory enthesitis. Four patients had an uncomplete response to NSAID at three months and requested high doses of NSAID (> $300 \mathrm{mg}$ Diclofenac or $>40 \mathrm{mg}$ Piroxicam). Two patients accepted methotrexate $7.5 \mathrm{mg}$ weekly with corticosteroids started at $15 \mathrm{mg}$ daily. A good clinical and biological response was observed after six weeks of treatment. Corticosteroids were reduced to $7 \mathrm{mg}$ a day, systemic symptoms were also controlled until their last visit in summer 1996. Two patients failed to respond to treatment with methotrexate and still require the combination of corticosteroids $15 \mathrm{mg}$ and NSAID with an incomplete response on pain at multiple sites, spine, anterior chest wall, and diffuse enthesitis of lower limbs. These two patients can be regarded has having a severe refractory presentation of late onset disease.

\section{Discussion}

Our results suggests that SPA as defined by accepted international criteria and with onset after 55 years (mean 65.1) differs, in clinical and biological presentation when compared with a group of patients with SPA beginning before 40 years. Upper inflammatory spinal pain (cervical and dorsal), anterior chest wall involvement, peripheral arthritis, aseptic osteitis, and systemic symptoms are significantly more frequent at presentation in older patients. A poor response to NSAID was observed in patients with inflammatory bowel disease who were successfully treated with prednisone.

The incidence rate of SPA after 55 years is $2.2 / 100000$ compared with $16.2 / 100000$ in the patients in the 25-34 years group. ${ }^{1}$

The overall frequency of SPA beginning after 50 years is estimated at $4 \%-8 \%$ in SPA population. $^{89}$

Calin compared the clinical expression of classic SPA between patients with classic age at onset (mean 22.6 years) and late onset (mean 38.7 year). The one difference was more shoulder pain in the late onset group. ${ }^{10}$

In patients described by Dubost and Sauvezie with peripheral seronegative spondylarthropathy, axial involvement is absent or mild, oligo-arthritis is associated with pitting oedema in a context of severe systemic symptoms. ${ }^{6}$ In the prospective study of patients with late onset undifferentiated spondylarthropathy reported by Olivieri, the clinical spectrum is found as wide as in children and middle age adults and include patients with pitting oedema. ${ }^{5}$ The male to female ratio of patients is 1:1. No association with inflammatory bowel disease or psoriasis was present. Interestingly most of these patients had raised ESR (mean $43.3 \mathrm{~mm}$ ).

There are evident limitations to our study. The sample size of late onset SPA is small. Only eight patients were seen in our department during eight years. Our study is a retrospective chart review with the inherent bias caused by this type of study. It is possible that the differences observed in LOSPA may result from practice setting-that is, only patients with unusual symptoms being referred to the department.

In conclusion, even though it is rare the diagnosis of SPA must be considered in female and male patients after 55 years.

We have found differences in clinical pattern between late onset inflammatory SPA and early onset SPA, which suggest that age may influence disease expressions of SPA.

Systematic evaluation of larger groups of patients are needed to confirm these preliminary data. 
1 Carbone LD, Cooper C, Michet CJ, Atkinson EJ, O'Fallon WM, Melton LJ. Ankylosing spondylitis in Rochester, Minn

2 Burgos-Vargas R, Naranjo A, Castillo J, Katona G. Ankylosing spondylitis in the mexical mestizo. Patterns of disease according to age at onset. J Rheumatol 1989;16:186-91.

3 Marks S, Bennett M, Calin A. The natural history of juvenile ankylosing spondylitis : a case control study of juvenile and adult onset disease. J Rheumatol 1982;9:73941.

4 Olivieri I, Padula A, Pierro A, Favaro L, Oranger GS, Ferri $S$. Late onset indifferentiated seronegative spondylarthropathy. J Rheumatol 1995;22:899-903.

5 Olivieri I. Oranges GS, Sconosciuto F, Padula A, Ruju GP, Pasero G. Late onset peripheral seronegative spondyloarthropathy. J Rheumatol 1993;22:390-3.
6 Dubost JJ, Sauvezie B. Late onset peripheral spondylarthropathy. J Rheumatol 1989;16: 214-7.

7 Amor B, Dougados M, Mijiyama M. Critères de classification des spondylarthropathies. Rev Rhum 1990;57:85-9.

8 Amor B, Dougados M, Listrat V, et al. Evaluation des critères des spondylarthropathies d'Amor et de l'European spondylarthropathy Study Group (ESSG). Une étude transversale de 2228 patients. Ann Med Interne 1991;142:85-9.

9 Wilkinson M, Bywaters EGL. Clinical features and course of ankylosing spondylitis as seen in a follow-up of 222 hospital referred cases. Ann Rheum Dis 1958;17:209-28.

10 Calin A, Elswood J, Edmunds L. Late onset ankylosing spondylitis. A distinct disorder? Br J Rheumatol 1991; 30:69-70. 